\title{
ECONOMÍA Y TRABAJO: LA GRAN TRANSFORMACIÓN DE LA SOCIEDAD ESPAÑOLA
}

\author{
Juan E. Iranzo
}

Instituto de Estudios Económicos

\begin{abstract}
RESUMEN
España se ha convertido una economía moderna, plenamente incorporada a la realidad económica global e integrada en un proyecto ambicioso como es el euro. La enorme transformación que hemos experimentado en los últimos cincuenta años es el resultado, por un lado, de la consolidación de nuestra apertura externa y, en especial, nuestra activa participación en el proceso de construcción europea y, por otro, de la estabilidad macroeconómica y el protagonismo del mercado, como mecanismo de asignación de recursos, frente al tradicional intervencionismo público, que caracterizó buena parte de nuestra historia económica reciente. En definitiva, el camino de la prosperidad, el crecimiento y la generación de riqueza se deriva de la integración en los mercados mundiales y, en particular, en el proyecto común europeo, con el fin de aprovechar los beneficios de la globalización sobre la productividad, la especialización, el mayor crecimiento y la creación de empleo.
\end{abstract}

En los albores del siglo XXI, nuestro país se ha convertido en una economía moderna, plenamente incorporada a la realidad económica global e integrada en un proyecto ambicioso como es el euro. La enorme transformación que hemos experimentado en los últimos cincuenta años no ha sido casual, sino que es el resultado de las decididas apuestas de la economía española: por un lado, la consolidación de nuestra apertura externa y, en especial, nuestra activa participación en el proceso de construcción europea y, por otro, la estabilidad macroeconómica y el protagonismo del mercado, como mecanismo de asigna- 
ción de recursos, frente al tradicional intervencionismo público, que ha caracterizado buena parte de nuestra historia económica reciente. $Y$ es que nuestra experiencia histórica en el siglo XX nos ha enseñado que el camino de la prosperidad, el crecimiento y la generación de riqueza no debía buscarse en el equivocado y costoso proteccionismo, sino en la integración en los mercados mundiales y, en particular, en el proyecto común europeo, culminado con el euro, con el fin de aprovechar los beneficios de la globalización, a través de sus efectos positivos sobre la productividad, la especialización, el mayor crecimiento y la creación de empleo.

\section{LOS AÑOS DE AUTARQUÍA Y RETRASO (1939-1959)}

El aislamiento internacional era una característica de nuestra economía ya desde la segunda mitad del siglo XIX. Tras una dura pugna entre defensores del librecambio y partidarios del proteccionismo, éste resultó definitivamente vencedor con el establecimiento del arancel de 1891, triunfando, de este modo, los intereses de la industria textil catalana, de los cerealistas castellanos y de la siderurgia vasca. Posteriormente, en 1906, se dicta la Ley de Bases Arancelarias (endurecida en 1922 por el arancel Cambó, y que se mantuvo hasta 1960), que, junto con las Leyes de Protección de la Industria Nacional de 1907 y 1917, condujeron al país a una situación de generalización de "prácticas monopolísticas, atraso tecnológico y raquitismo industrial» (Tamames, 1982). El derrumbe de los mercados mundiales, tras la Gran Depresión del período de entreguerras, no favoreció el cambio hacia una mayor apertura de la economía. $\mathrm{Al}$ contrario, comenzaron a aplicarse otros instrumentos proteccionistas que sustituyeron o complementaron a la política arancelaria (controles de cambio y contingentes). Los Gobiernos republicanos se encontraron, a continuación, sobrepasados por la creciente conflictividad interna, que conduciría inexorablemente al desastre de la Guerra Civil (González, 1979).

Una vez concluida la contienda, distintos factores dificultaron la recuperación económica, así como el aprovechamiento de las posibilidades comerciales que ofrecía la II Guerra Mundial a una nación neutral como la nuestra: por una parte, el aparato productivo nacional se encontraba seriamente dañado $y$, por otra, se añadía la dificultad de entablar relaciones comerciales con las economías europeas en guerra, así como la carencia de medios de pago internacionales. A ello se une el bloqueo internacional a partir de 1946 por nuestro apoyo a las potencias del Eje, con lo que se esfumaba cualquier posible alternativa en materia de política económica. Por tanto, el período autárquico de la economía española (1939-1959) se desarrolló en un entorno internacional adverso. Es decir, se adoptó un modelo excepcional, fuertemente proteccionista y basado en una indiscriminada sustitución de importaciones, con el fin de reconstruir un país destruido por nuestra contienda y lograr un alto grado de autoabastecimiento. Pero, al mismo tiempo, se puede hablar de la consuma- 
ción de tendencias anteriores, ya existentes en la política económica española. El Estado no hizo sino mantener y fortalecer la política proteccionista y de fomento industrial anterior, que reflejaba la tradicional postura de una amplia burguesía industrial y financiera, interesada en limitar o, incluso, eliminar la competencia externa.

No obstante, el modelo autárquico no fue del todo impuesto por las circunstancias externas (si bien éstas también son, en cierto modo, consecuencia de la opción política adoptada por el régimen), sino que fue puesto en práctica, en parte, por convicción ideológica y una cierta necesidad legitimadora: la idea del nacionalismo autosuficiente, muy relacionado con elementos aislacionistas, y común a todos los regímenes de ideología similar, surge como la única opción válida para reconstruir el país (Prados de la Escosura, 1997). Asimismo, se desconfía del mercado y se ensalza, en su lugar, el papel del Estado como órgano regulador de la actividad e impulsor de los intereses nacionales, de lo que se deriva el fomento sólo de aquellas industrias que el régimen consideraba de interés nacional (Comín, 1993a, y Segura, 1993). En este sentido, la política proteccionista se puso en práctica con una amplitud e intensidad muy superiores y fue acompañada de un extenso intervencionismo, que pretendió reglamentar la práctica totalidad de las actividades económicas (Comín, 1993b).

El modelo autárquico era contradictorio con algunas leyes económicas fundamentales, lo que conducía a reemplazar los criterios basados en la eficacia económica por un intervencionismo autoritario y arbitrario, caracterizado por un ingenierismo de signo militar, donde predominan los criterios de carácter técnico por encima de consideraciones de tipo económico (Velasco, 1984, y Comín, 1996). Todo ello, además, a costa de ignorar las limitaciones del modelo, que se encuentra con tres obstáculos principales. En primer lugar, la escasez, tanto de materias primas como de bienes de capital o de una tecnología adecuada, en una economía destruida por la guerra, lo que explica, en contra del deseo de las autoridades, la creciente dependencia del exterior. Esto justifica la adopción de una producción autóctona más costosa, con el fin de satisfacer una demanda ampliamente insatisfecha, en una situación de carestía generalizada: el caso del gasógeno es un ejemplo, ante la escasez de gasolina. En segundo lugar, el tamaño limitado del mercado interior impedía el aprovechamiento de economías de escala (Fuentes, 1993), así como alcanzar un elevado crecimiento, lo que implica que se renuncia a generar divisas a través de las exportaciones (Carreras, 1997). Por último, la tercera limitación es que el intervencionismo y la protección frente a la competencia exterior niegan las ventajas del comercio y la especialización internacional, lo que da lugar, por un lado, a una producción de muy baja calidad con unos costes muy elevados (industrias poco competitivas y rentables) y, por otro, a un amplio aparato burocrático y administrativo que, en lugar de incentivar la producción de calidad y la inversión, anima la aparición de grupos de interés en busca de favores (licencias, permisos, cupos, mayor protección, etc.) entre las instancias administrativas (Requeijo, 1990). 
Precisamente, la característica diferenciadora de la autarquía española no radicaba en el intervencionismo en sí mismo, sino en el carácter de permanencia del modelo, por una parte, y en la incapacidad del aparato administrativo para efectuar los controles necesarios, que, junto a la escasez, se tradujo en la aparición de actitudes corruptas y de un vasto mercado negro, como respuesta a la excesiva reglamentación (Barciela, 1994). Los efectos más graves para el desarrollo a largo plazo fueron la limitación y el desincentivo de la actividad empresarial y de la iniciativa privada, junto a la dilatación en el tiempo de actitudes equivocadas y erróneas por parte de la Administración, al contar con tanto margen de actuación en un entorno protegido de no mercado (Comín, 1993b). Pero será el propio mecanismo del modelo de crecimiento autárquico el que provocará su desplome a finales de la década de los cincuenta (Tamames, 1982), puesto que el proceso industrializador, basado en un fuerte proteccionismo, junto a una agricultura tradicional y postergada, necesariamente debía conducir a la aparición de tensiones. Cuando a partir de 1953 (año en que se recuperaron los niveles de renta de antes de la Guerra Civil) se afianza la recuperación definitiva de la economía (en la década de los cincuenta el PIB crece entre un 3,5 y un 5 por 100 anual, frente a un incremento de entre el 1,2 y 1,9 por 100 en la década anterior), comienzan a hacerse patentes las limitaciones del modelo (Martín Rodríguez, 1993, y Carreras, 1987).

El mayor dinamismo de la economía corresponde, sin duda, al sector industrial, gracias a la lenta eliminación de obstáculos y trabas administrativas y a las mayores facilidades para importar equipo y materias primas, ya que las mejores cosechas reducen nuestra necesidad de importar alimentos y se cuenta con las ayudas y créditos extranjeros. No obstante, las mayores necesidades de este crecimiento (medios de producción y bienes de equipo) chocan con una política excesivamente intervencionista que restringe forzosamente el ritmo de recuperación (González, 1979). La pretendida autosuficiencia se convierte, así, en una utopía irrealizable, por factores internos y externos. En primer lugar, se generó un sector industrial fuertemente protegido y, por tanto, escasamente competitivo, con unos costes de producción crecientes y una notoria ineficiencia. En este proceso jugó un papel principal el Estado, quien intervino de forma directa en la actividad productiva, por medio de costosas inversiones. Este elevado volumen de gasto público fue financiado de forma poco ortodoxa mediante la monetización de los déficit públicos, la llamada "política de inflación e industrialización» (Fuentes, 1984, y Aríztegui, 1993). En consecuencia, la elevada inflación que caracterizó a la economía española no sólo se debió a la rigidez del aparato productivo y a la consecuente escasez reinante en el mercado interior, que no fue complementada por las importaciones, sino también a la ausencia de una verdadera disciplina monetaria. Sin embargo, la vía fiscal no fue una verdadera alternativa de financiación pública, puesto que la capacidad recaudatoria del sistema impositivo era muy limitada, debido al fraude generalizado en la época y a un sistema fiscal anticuado y francamente ineficiente (Barciela, 1994, y Portillo, 1998). Por tanto, se puede hablar de una 
política económica de posguerra acomodante, de incremento de las disponibilidades monetarias y del gasto público, y que no hizo sino agudizar el tradicional círculo vicioso de nuestra economía: inflación, depreciación, protección y más inflación (Varela, 1990).

Por otra parte, la baja productividad en el sector agrario, como consecuencia de la menor dotación de capital y de la abundante mano de obra existente, fue, en buena medida, el factor explicativo del tradicional atraso de nuestra economía (Prados de la Escosura, 1997). España se encuentra, así, en un círculo vicioso, pues la incipiente industrialización, dada la estrechez del mercado nacional, precisaba de una agricultura moderna con alto poder adquisitivo o, en su defecto, de centros urbanos capaces de absorber la excesiva mano de obra agraria, en cuyo caso los rendimientos de la industria se ven frenados por la limitación que supone el tamaño del mercado (Perpiñá, 1993). Precisamente, los mayores índices de productividad (y de salarios) que comienzan a registrarse en la industria y en los servicios provocan las primeras oleadas del éxodo rural hacia los núcleos urbanos y desencadenan la crisis de la agricultura tradicional (Carreras, 1987, y Velarde, 1996). A esto hay que añadir un hecho concreto y desafortunado que incidió también sobre la demanda (González, 1977): las subidas salariales decretadas en marzo y octubre de 1956 por el Ministro de Trabajo, José Antonio Girón de Velasco. Los precios agrarios se vieron, así, presionados al alza, dado que el sector se encontraba en una situación "estacionaria» desde antes de la Guerra y era incapaz de responder al incremento de demanda (Perpiñá, 1993).

Por lo que respecta al factor externo, la dependencia exterior, en lugar de disminuir, se incrementó: aumentaron de forma notable las importaciones de bienes de equipo (el 37 por 100 del total en 1957), inputs o carburantes, indispensables para proseguir con el desarrollo industrial (Fanjul, 1981, y Serrano, 1997). Estas importaciones fueron financiadas, en un primer momento, con productos agrarios, principalmente. Sin embargo, la escasez y limitada diversificación de estas exportaciones, junto con su dependencia de las condiciones climáticas y la evolución de las cosechas, condicionan enormemente nuestro desarrollo industrial. Nuestra incapacidad exportadora se ve agravada, además, por unos tipos de cambio artificiales y arbitrarios que incentivan la importación y la salida de capitales y por nuestros elevados precios internos. En estas circunstancias, no puede menospreciarse la ayuda norteamericana que, entre 1951 y 1957, permitió una cobertura en divisas de más del 15 por 100 del total de importaciones (Fanjul, 1981), aunque ello no impidió tener que recurrir a nuestras escasas reservas de oro. A pesar de todo, nuestro déficit comercial continuó ampliándose de forma incesante hasta 1958, año en que la situación se hace insostenible por la falta de divisas. El factor externo es el que desencadena la crisis, pues el gran error fue creer que el rápido crecimiento de los años cincuenta podría mantenerse en ausencia de un comercio exterior liberalizado y un tipo de cambio razonable (Requeijo, 1990, y Núñez, 1998). Se cumple, de este modo, la primera ley de la política económica española, según 
la cual sólo ante una situación exterior como la descrita se adoptan las medidas adecuadas.

Por tanto, se puede afirmar que esta paulatina política liberalizadora no fue el resultado de ninguna estrategia, sino que fue consecuencia de las propias trabas que estrangulaban nuestro crecimiento, lo que supone el reconocimiento del fracaso de la opción autárquica. La falta de coordinación administrativa y la parcialidad de las medidas pre-estabilizadoras de 1957 (subida del tipo de redescuento bancario y reducción del volumen de crédito, junto con la unificación del tipo de cambio) hicieron fracasar esos intentos (González, 1979), puesto que no se frenó la inflación, al seguir permitiendo el recurso al Banco de España para financiar los desequilibrios presupuestarios. Sin embargo, estas medidas constituyeron un primer paso en aras de eliminar los desequilibrios internos que atenazaban nuestra economía (déficit público e inflación), aunque, a cambio, se amplió nuestro desequilibrio externo. Por otra parte, tuvieron cierta influencia en este lento cambio de actitud determinados acontecimientos externos, como la firma del Tratado de Roma en 1957 o nuestra incorporación a los organismos económicos internacionales (FMI, Banco Mundial y OECE), lo que nos permitió acceder a importante ayuda técnica y financiera. Se hacía evidente, por fin, la imposibilidad de mantenernos al margen de las pautas de crecimiento reinantes en las economías internacionales, por lo que se vislumbran dos alternativas: aceptar un agudo descenso del nivel de vida o acometer el Plan Nacional de Estabilización Económica de 1959, que conducirá a una cierta liberalización, tanto del mercado interior como del comercio exterior, y supone el comienzo de una nueva estrategia de política económica.

\section{EL PLAN DE ESTABILIZACIÓN Y LOS AÑOS DE DESARROLLO (1959-1973)}

El Plan de Estabilización se basó en tres pilares (Fuentes, 1993): en primer lugar, la liberalización del comercio exterior, facilitando los intercambios mediante la convertibilidad de la peseta, lo que permitió disfrutar de la bonanza de la economía internacional, con el consiguiente aumento del nivel de vida. Al mismo tiempo, también se incrementaron las importaciones de bienes de equipo, lo que permitió el acceso a la tecnología puntera, y se revisó la Ley de Inversiones Exteriores para facilitar la llegada de ahorro exterior. En segundo lugar, la estabilización interna, que supone el abandono de la política del "crecimiento inflacionario". Con este fin, en el sector público se introducen limitaciones al gasto total y a la emisión de deuda y se suprimen los subsidios a empresas públicas, mientras que en el sector privado se suben los tipos de interés y se limita el crecimiento del crédito y de las operaciones activas de la banca. Por último, la mejora de la asignación de recursos, a través de la mayor competencia en el mercado interior. En esta línea, se suavizan los requisitos 
para el establecimiento de nuevas empresas y se dictan dos Leyes en 1963: la Ley de Prácticas Restrictivas de la Competencia y la Ley de Industrias de Interés Preferente, que suavizó las Leyes industriales de 1939 y sus estrictos controles sobre las industrias llamadas de "interés nacional». En consecuencia, desde 1960 hasta la primera crisis energética de 1973, España disfrutó de un período de crecimiento sin precedentes, gracias a la favorable coyuntura internacional $y$, en concreto, los bajos precios de la energía, una tecnología accesible, la amplia entrada de capital (turismo, emigrantes e inversores que compensaban el déficit comercial) y una mano de obra abundante (García Delgado y Jiménez, 1999).

El sector industrial fue, sin duda, el impulsor de este auge, por lo que la economía española, por fin, experimentó, aunque de forma tardía, una fase de industrialización y tecnificación de su tejido productivo (Tortella, 1995). La industria creció muy por encima de la economía en su conjunto (10,4 frente a 6,2 por 100 anual en el período 1958-1972), de tal forma que en 1974 representaba el 34 por 100 del PIB (ocho puntos más que diez años antes). Por su parte, la población ocupada en el sector secundario tan sólo ganó, en el mismo período, un punto porcentual hasta el 26 por 100 del total, lo que significa un importante aumento de productividad. El sector industrial, por otro lado, se benefició de la apertura exterior de la economía, pues las exportaciones industriales aumentaron, en la década de los sesenta, por encima del 14 por 100 de media anual. Destaca especialmente la industria del automóvil, con un crecimiento medio anual cercano al 22 por 100 entre 1958 y 1972 (Donges, 1976), que, a su vez, tiró con fuerza del sector del caucho (neumáticos), siderúrgico y del petróleo. El tirón del automóvil puso de relieve la capacidad de nuestra industria para desarrollar una actividad totalmente nueva en aquel momento, así como el aumento del nivel de vida, dado que la práctica totalidad de la producción se vendía en el mercado interior, y ello a pesar de sus elevados precios. Otros sectores que se vieron impulsados en este período fueron la construcción naval, la siderurgia o la minería, industrias con un futuro incierto, elevada intensidad en mano de obra y tecnología poco avanzada, y que posteriormente, debido a la crisis económica de los años setenta, protagonizarían una necesaria y, al mismo tiempo, costosa reconversión.

Aunque con el Plan de Estabilización y las medidas que le siguieron se dio un paso muy importante hacia la liberalización de la economía, lo cierto es que los cambios fueron lentos y permanecieron algunas restricciones, un cierto proteccionismo y elementos monopolísticos. Así, el INI siguió jugando un papel clave en la política industrial y el sistema autárquico fue sustituido por otro tipo de intervencionismo, a través de los Planes de Desarrollo que se inician en 1964, y que, en parte, desvirtúan el espíritu liberalizador del Plan de Estabilización, al desequilibrar el crecimiento y desperdiciar importantes oportunidades de expansión (Fuentes, 1993). En 1963 se vuelve a introducir el sistema de requisitos y licencias para el establecimiento de nuevas empresas, con el fin de evitar el minifundismo empresarial, pero que no hacía sino perpetuar los privi- 
legios de la vieja industria establecida (González, 1979). En cuanto al comercio exterior, los contingentes fueron sustituidos por el arancel de 1960 y se crearon los créditos especiales a la exportación o las llamadas "cartas de exportador», de tres categorías distintas (Requeijo, 1983). El sistema de privilegios del intervencionismo desarrollista entra en crisis tras el "escándalo MATESA», empresa de telares que, exportando a sus propias sucursales en otros países, pudo sortear los correspondientes controles y beneficiarse de los créditos a la exportación, que eran gestionados por el Banco de Crédito Industrial. Este caso puso al descubierto la incompetencia del régimen para gestionar, mediante toda una serie de controles burocráticos, una economía de mercado, puesto que lo realmente importante en la asignación de los recursos era el favor político y el amiguismo.

\section{LA CRISIS DE LOS AÑOS SETENTA Y LA TRANSICIÓN A LA DEMOCRACIA (1973-1985)}

A partir de entonces, y hasta el final del régimen franquista, puede hablarse de una «segunda transición hacia el liberalismo" (Tortella, 1995), pero igualmente lenta e inconclusa, por el temor de las autoridades a las consecuencias políticas de una mayor libertad económica. La ausencia de un programa económico adecuado dificultó, sin duda, la salida de la crisis económica internacional de 1973, que, en el caso español, se saldó con una importante destrucción de empleo y de tejido productivo, especialmente en el sector industrial (que, entre 1978 y 1984, registró una contracción del 2,2 por 100), como consecuencia, por un lado, de la ya señalada especialización en sectores intensivos en factor trabajo y escasa tecnología y, por otro, de la baja competitividad derivada del tradicional marco proteccionista en que se desenvolvía. En estas circunstancias, se hacía imprescindible una política de reconversión industrial, mediante la transferencia de recursos desde actividades poco rentables a otras más competitivas. Sin embargo, estas medidas, impopulares pero necesarias, se pospusieron, prolongando, en consecuencia, la situación de crisis.

Nuestro país se vio perjudicado, al igual que otros países de su entorno, por el encarecimiento del petróleo, si bien nuestra mayor dependencia energética y la errónea política compensatoria llevada a cabo, por la que los aumentos del precio del crudo eran absorbidos íntegramente por el Estado sin repercutir en los consumidores, agravaron notablemente sus consecuencias. El consumo energético no se contuvo y el déficit por cuenta corriente se disparó, lo que obligó a rectificar, trasladando los mayores precios energéticos a los consumidores, pero estableciendo, a cambio, una política monetaria restrictiva (con el fin de limitar las tensiones inflacionistas) que, a la postre, tuvo efectos nocivos sobre la actividad, con caídas de la inversión, estancamiento del consumo y de la producción y aumento del desempleo. La inflación, asimismo, se vio impulsada por las dos devaluaciones de febrero de 1976 y julio de 1977. Pero, además, se añaden una serie de factores propios derivados de la transición a la 
democracia. Por un lado, la incertidumbre y la desconfianza empresarial en el nuevo marco institucional explican parte del retroceso de la inversión en estos años. Por otro, la economía se encuentra con algunos lastres heredados de la época franquista (García Delgado y Jiménez, 1999): la escasa eficiencia del sector financiero, una regulación laboral «autoritaria y paternalista» (Núñez, 1998), que aseguraba el empleo pero limitaba, a cambio, la libertad de los trabajadores, y un sector público que, pese a su reducido tamaño, contaba con excesivas dosis de intervencionismo en la economía.

El régimen democrático recién estrenado se enfrentaba, por tanto, a graves problemas políticos, sociales y económicos que podían acabar erosionando su legitimación. De este modo, en octubre de 1977 se firman los llamados Pactos de La Moncloa, suscritos por todos los partidos políticos con representación parlamentaria y que permitieron crear la sensibilidad adecuada para afrontar con responsabilidad la grave crisis económica. Por vez primera en nuestra historia, la política económica dispuso de una base, acordada por amplio consenso, para la aplicación de un programa que afrontaba los acuciantes problemas que comprometían nuestro futuro. Se requerían importantes esfuerzos para moderar nuestra galopante inflación (cercana al 45 por 100 en los meses centrales de 1977), que lastraba la competitividad internacional de nuestras empresas, y frenar un endeudamiento externo insostenible que acabaría hipotecando el futuro económico del país (nuestro déficit por cuenta corriente superaba por aquel entonces los 5.000 millones de dólares) (Requeijo, 1988). En consecuencia, había que sanear la economía y aplicar una serie de reformas y ajustes, había que alcanzar los equilibrios económicos necesarios, en términos de precios y balanza por cuenta corriente, para combatir el reducido crecimiento y el elevado desempleo.

En cuanto al programa de saneamiento, se toma conciencia de que la lucha contra la inflación es, más que una opción de política económica, una cuestión de supervivencia para la sociedad. Esta política antiinflacionista debía apoyarse en cuatro pilares. En primer lugar, una política monetaria restrictiva, basada en el control de la cantidad de dinero en circulación. En segundo lugar, una política presupuestaria de contención del gasto público, en especial el de naturaleza corriente. Tercero, una política cambiaria realista con el fin de orientar las corrientes de comercio exterior. Y, por último, una política de rentas basada en cuatro aspectos: el incremento salarial en función de la inflación prevista (y no la inflación histórica); la reducción de las cuotas de la Seguridad Social sobre los salarios; la moderación de los costes financieros, por medio de la liberalización y apertura exterior del sistema financiero, y la liberalización de los mercados de bienes y servicios. Por lo que se refiere a las medidas de reforma, se incluyeron cinco líneas de acción fundamentales: el presupuesto y el gasto público, con el fin de introducir mayor disciplina; la anticuada estructura tributaria; el sistema financiero; un nuevo marco de relaciones laborales, y el papel de la empresa pública. También se contemplaron algunas reformas encaminadas a modernizar nuestra estructura productiva y favorecer una mejor uti- 
lización de los recursos del país. Así, por ejemplo, el Plan Energético Nacional de 1978 supuso la primera aproximación a una política energética realista, mediante el ajuste de los precios interiores a los costes reales de los productos energéticos importados o una política más coherente de incentivos para el ahorro y la diversificación de fuentes.

Los efectos positivos de los Pactos de La Moncloa fueron tanto económicos como políticos. Por un lado, la inflación cambió su tendencia alcista y concluyó 1978 en el 16 por 100, se recuperó el equilibrio de la balanza por cuenta corriente (con superávit en 1978 y 1979) y se sanearon las maltrechas finanzas de las empresas, lo que permitiría impulsar, posteriormente, la inversión privada. Por otro lado, también se produjo un importante cambio en el clima político del país, en tanto que este proceso de estabilización, de forma ordenada y sin traumas, desembocó en la promulgación de la Constitución de 1978. Por su parte, la crisis de la industria obligaba a acelerar su apertura al exterior y, en consecuencia, a afrontar la necesaria reconversión, esto es, una serie de cambios en su organización y en la calidad y características de sus productos que le permitiera enfrentarse con éxito a la competencia internacional. Así, en 1981 y 1982 se dictaron un Decreto-Ley y una Ley, respectivamente, de reconversión de algunos sectores e industrias, pero con escaso éxito.

Es a partir de 1982 cuando comienza a aplicarse una política económica más estructurada y coherente (Tortella, 1995), con especial atención en materia de reconversión. En este sentido, la Ley de Reconversión y Reindustrialización de 1984 creó las llamadas «zonas de urgente reindustrialización» e incluía créditos y subvenciones a nuevas inversiones y planes de jubilación anticipada. El INI fue obligado también a aplicar estos principios, mediante la reducción de su plantilla, la aceleración de las amortizaciones y la racionalización de la inversión. No obstante, aumentó la conflictividad laboral, como respuesta a los severos ajustes de plantillas que, en algunos casos, debieron acometerse. Al éxito de esta política (a partir de 1985 la inversión y la producción industrial registraron una notable mejoría) contribuyó, sin duda, la recuperación de la economía mundial, la normalización de los precios del petróleo y nuestra inminente integración en la Comunidad Europea (CE), cuyo Tratado de Adhesión, después de un largo proceso negociador, se firmó en junio de 1985.

\section{EL IMPULSO DE LA INTEGRACIÓN EN EUROPA (1985-1992)}

La integración en la CE potenció fuertemente nuestro comercio exterior, en el corto plazo, aunque no siempre de forma positiva (creación y ampliación de comercio), ya que también surgió el perverso fenómeno de la desviación de una parte importante del comercio con terceros países hacia las naciones comunitarias. Así, los intercambios con la CE, que representaban en torno al 50 por 100 de nuestras exportaciones y tan sólo un tercio de las importaciones, se elevaron hasta alcanzar a mediados de los años noventa cotas del $70 \mathrm{y}$ 
del 60 por 100 , respectivamente. Al mismo tiempo, el crecimiento de nuestras compras al exterior fue considerablemente mayor al de las ventas, lo que redujo significativamente la tasa de cobertura y provocó un deterioro notable del saldo comercial con la CE, poniendo, así, en evidencia algunas de las debilidades estructurales de nuestra economía. Y es que el modelo de crecimiento aplicado durante la fase expansiva de finales de los años ochenta se sustentó en la fortaleza de la demanda interna, cuyo ritmo de aumento duplicaba las tasas medias de variación de los demás países de la UE. Además, la expansión de la economía mundial, y en particular de la economía europea, actuaba de locomotora de nuestro crecimiento, en un contexto claramente favorecido por la contención de los precios de las materias primas (Requeijo, 2000).

$\mathrm{El}$ intenso dinamismo del gasto interior hizo que una parte del mismo se filtrara al exterior, tanto por la vía del incremento de las importaciones (dado que la oferta nacional resultaba insuficiente) como por la menor progresión de las exportaciones. En este contexto de bonanza interior, las empresas españolas no necesitaban dedicar grandes esfuerzos a la siempre difícil tarea de abrirse paso en los mercados internacionales. Además, la incorporación de España a la CE implicó el desmantelamiento de nuestra secular estructura proteccionista, con la supresión de las barreras arancelarias que entorpecían los flujos comerciales con los países comunitarios, mientras que la apreciación del tipo de cambio efectivo real de la peseta deterioró gravemente la competitividad de nuestras empresas.

Los mayores beneficios para España, derivados de la integración europea, han sido los ligados a la transformación estructural fruto de la misma. Fue especialmente destacable la capitalización de nuestra economía en la segunda mitad de los años ochenta, ayudada por la afluencia masiva de inversión extranjera. El proceso inversor se debió, sobre todo, al esfuerzo realizado por los empresarios españoles para modernizar sus estructuras productivas, a fin de poder enfrentarse con mayores garantías a un entorno más competitivo. No obstante, las empresas no habrían podido emprender nuevos proyectos de inversión de no haberse producido un notable saneamiento de sus posiciones financieras. En este sentido, la recuperación de los márgenes empresariales permitió la liberación de rentas, que pudieron canalizarse hacia la renovación y ampliación de la capacidad instalada. Asimismo, la gravedad del desempleo y la flexibilización de la entrada al mercado de trabajo mediante el establecimiento de las modalidades de contratación temporal en 1984 posibilitaron una importante moderación de los salarios y, en consecuencia, la disminución de los costes laborales unitarios hasta niveles inferiores a los de nuestros socios comerciales. El problema es que, a cambio, se generó una presión excesiva sobre el gasto público en la cobertura de desempleo, prestación que distorsionaba el correcto funcionamiento del mercado de trabajo (elevando los salarios de reserva).

El Gobierno, a los efectos de ayudar a las empresas en su preparación al reto europeo, otorgó, a mediados de los años ochenta, un paquete de incenti- 
vos fiscales, entre los que destacaba la libertad de amortización. Esta medida redujo el coste del capital a través de la disminución de su componente fiscal y contribuyó a compensar el efecto negativo de los altos tipos de interés vigentes en aquellos años. Además, la aprobación de algunas medidas liberalizadoras, como la libertad de horarios comerciales o la reforma de la Ley de Arrendamientos Urbanos, ayudó a moderar la tasa de inflación hasta situarla en el 4,5 por 100 en 1988, el nivel más bajo desde 1975. La desaceleración de los precios supuso, a su vez, un fuerte estímulo para la mejora de las expectativas empresariales. En este sentido, la reducción del déficit presupuestario entre 1985 y 1988, resultante del efecto de los estabilizadores automáticos, también contribuyó a mejorar la opinión de los empresarios sobre la coyuntura, en la medida en que abría la posibilidad de descensos de los tipos de interés. No obstante, se desaprovechó aquel período de bonanza para atajar el componente estructural del déficit, con la excepción de la reforma de las pensiones de 1985. En este sentido, el déficit público estructural rondaba el 6 por 100 del PIB en 1991, frente al 1,3 por 100 en que se situó entre 1978 y 1982. De esta forma, la política fiscal, tras la contención del déficit que terminó en 1988, empezó a caracterizarse por un tono inmoderadamente procíclico cuando más contraproducente era. De hecho, el Gobierno, tras la huelga general de 1988, comenzó a ceder a las presiones sindicales incrementando el gasto público sin control. Por ello, una vez que se agotó el ciclo expansivo, el déficit público volvió a repuntar con fuerza desde 1989 hasta alcanzar su máximo en 1993 (7,5 por 100 del PIB), cuando la dureza de la recesión disminuyó los ingresos públicos y disparó el gasto asignado a los programas públicos. El carácter expansivo de la política fiscal fue uno de los grandes responsables de la generación de tensiones inflacionarias y de la elevación de los tipos de interés en España.

La inversión extranjera acudía con gran intensidad, con la finalidad de aprovechar la generación de las plusvalías derivadas de nuestra entrada en la CE. Además, otras naciones industrializadas optaron por materializar parte de sus procesos productivos en nuestro país, al que consideraban una plataforma idónea para tener acceso a todo el mercado comunitario. Las fuertes expectativas de crecimiento hacían especialmente atractivas para los extranjeros tanto la compra de inmuebles como la de participaciones en empresas productivas, aunque más tarde se fue desviando hacia la compra de activos financieros (primero acciones y luego deuda pública). De hecho, desde principios de los años noventa la financiación de nuestro desequilibrio exterior descansó, de forma creciente, en la apelación al crédito exterior y en la compra de deuda pública por parte de no residentes. $\mathrm{Y}$ es que el deterioro de las finanzas públicas a finales de los años ochenta hizo ineludible el recurso al ahorro externo para financiarse. En consecuencia, el Gobierno se vio obligado a elevar los tipos de interés reales de la deuda pública y a mejorar el tratamiento fiscal de los títulos públicos en manos de no residentes, que quedaron libres de gravamen.

Otro fenómeno destacable fue el fuerte aumento de la competencia que 
supuso la incorporación de España a la CE, sobre todo en el sector industrial, cuyos efectos positivos para los consumidores españoles se dejaron sentir, tanto por la vía de los precios como de las calidades de los productos. Sin embargo, es muy posible que la competencia que sufrió el sector industrial fuese excesiva debido, en gran medida, a la apreciación artificial del tipo de cambio de la peseta en la segunda mitad de los años ochenta. En este sentido, la entrada de flujos de capital del exterior, en un primer momento, y el endurecimiento de la política monetaria, posteriormente, elevaron el precio relativo de nuestra moneda, que, aun cuando tuvo un efecto positivo sobre la inflación, destruyó una parte de nuestra capacidad instalada, en especial en los sectores abiertos a la competencia. La incorporación de la divisa española al Sistema Monetario Europeo (SME) en junio de 1989 agravó aún más la situación, en la medida en que generó expectativas positivas en los agentes económicos y, en especial, entre los inversores institucionales respecto a la conducción de la política económica, y ello aunque se ancló la peseta en una posición de tipo de cambio excesivamente apreciada desde el punto de vista de nuestras cuentas exteriores, lo que implicaba dos cosas. Por una parte, las autoridades españolas renunciaban a la utilización del tipo de cambio como mecanismo de ajuste automático para corregir los desequilibrios internos (inflación) y externos (déficit exterior) y se comprometía a solucionar estos problemas a través de la adopción de otras medidas que actuaran directamente sobre el funcionamiento de los mercados.

Por otra parte, dado que la política monetaria debía velar por la estabilidad cambiaria (aunque sin perder de vista su objetivo más importante, esto es, el control de los precios), se creía que la política presupuestaria y la de rentas se moderarían suficientemente (recortando el gasto y el déficit y ligando los incrementos salariales a la evolución de la productividad), tomando el relevo de la política monetaria en la lucha contra la inflación. Sin embargo, las distintas ramas de la política económica no se combinaron de forma adecuada a la nueva situación. Así, la política fiscal expansiva, que se aceleró desde la huelga general de 1988, y el fuerte crecimiento salarial registrado entre 1987 y 1990 dejaron toda la responsabilidad de la lucha contra la inflación a una huérfana política monetaria, que dio como fruto elevados tipos de interés, apreciación artificial de la peseta, limitación al crecimiento del crédito bancario y el establecimiento de un coeficiente para la disposición de créditos en divisas. Las restricciones monetarias aprobadas en 1989 tuvieron un impacto muy negativo sobre la economía productiva, especialmente sobre el sector industrial, ya que provocaron, por un lado, un fuerte crecimiento de los costes financieros de las empresas y, por otro, graves dificultades a los sectores exportadores, lo que se tradujo en una importante caída del excedente empresarial en el sector industrial. 


\section{LA CRISIS DE 1992-1993}

A comienzos de 1992, coincidiendo en el tiempo con las turbulencias del SME, se inicia formalmente la recesión, cuyos efectos se agravaron por la pasividad e incoherencia de la política económica de la época. Las tres grandes devaluaciones de la peseta entre septiembre de 1992 y mayo de 1993 manifestaron la quiebra definitiva de un esquema de política económica que, en ausencia del resto de armas, utilizaba la cotización sobrevalorada de la peseta y la política monetaria como medios para disciplinar la economía. La timidez de las devaluaciones y la ausencia de un acompañamiento ortodoxo de la política fiscal y de rentas generaban unas expectativas inflacionarias y de sucesivas devaluaciones de nuestra moneda que obligaban a pagar elevadas primas de riesgo (tipos de interés) a los inversores por sus posiciones en activos denominados en pesetas, ya que nuestra moneda perdió entre 1992 y 1993 un 20 por 100 de su valor frente a las monedas europeas y un 30 por 100 frente al dólar y al yen.

Por otra parte, la buena evolución del empleo en la segunda mitad de los años ochenta, la confrontación sindical, que llegó a su máximo con la huelga general de diciembre de 1988, y la pérdida de confianza en los objetivos y previsiones inflacionarios del Gobierno provocaron una fuerte aceleración salarial, que pasó del 5,5 por 100 en 1989 a casi el 9 por 100 en media anual en los tres siguientes ejercicios. De este modo, los salarios crecieron por encima de la productividad del trabajo y las rentas públicas se indiciaron con la inflación pasada, mecanismo completamente perverso que levantó una espiral precios-salarios de difícil contención. La rigidez excesiva del mercado laboral hizo el resto: la remuneración salarial siguió aumentando hasta 1993 sin tener en cuenta la intensa destrucción de empleo que se estaba produciendo, como consecuencia de la fuerte segmentación del mercado laboral entre trabajadores temporales (sobre los que recaía el ajuste) e indefinidos (que disfrutaban de las mejoras salariales obtenidas en los convenios al gozar de una mayor representación sindical). Hubo que esperar a que el ajuste del empleo empezara a afectar a los trabajadores indefinidos para que el mercado laboral comenzara a sensibilizarse de la delicada coyuntura que estaba viviendo. A ello también ayudó el hecho de que en 1994 se aprobara una reforma laboral que intentaba potenciar el desarrollo de la negociación colectiva como elemento regulador de las relaciones laborales y las condiciones de trabajo. Previamente, en el RD-L 18/1993 se había abierto la puerta a las agencias de colocación y a las empresas de trabajo temporal.

Además, en un primer momento, para evitar que el desbordamiento del déficit público superara en exceso los compromisos asumidos en el Programa de Convergencia y se elevara aún más la prima de riesgo, el Gobierno tuvo que actuar de forma precipitada y desafortunada, aprobando un plan de medidas presupuestarias urgentes. El problema es que, en lugar de actuar decididamente sobre el gasto corriente, optó por la vía fácil y recortó los gastos de capital, subió con carácter retroactivo los tipos del IRPF, las retenciones del trabajo 
personal y el tipo general del IVA. Sin duda, todas estas medidas, al ir justo en la dirección contraria de lo que las circunstancias aconsejaban, alimentaron el proceso de recesión que entonces comenzaba, sobre todo cuando, a partir de ese momento, se pospusieron las reformas estructurales de mejora de la competitividad que nos hubieran ayudado a sortear la crisis, con algunas excepciones de difícil justificación social, como fue el recorte de las prestaciones por desempleo cuando éste más acuciaba.

La causa del desequilibrio público se encontraba, sobre todo, en la explosión del gasto y en el agotamiento de un sistema fiscal cuya voracidad recaudatoria y excesivos tipos, además de estimular el fraude fiscal, fagocitaba la actividad y el empleo, cercenando sus principales bases imponibles, sobre todo cuando éstas descansaban, en exceso, en la fiscalidad empresarial (cotizaciones sociales e impuestos sobre sociedades). A este respecto, el ahorro nacional pasó de representar el 21,6 por 100 del PIB en 1986 a sólo el 19,4 por 100 en 1992, como consecuencia de la pérdida de capacidad de ahorro de las empresas (por la caída de sus resultados derivada del aumento de sus costes) y de los hogares (por la excesiva presión fiscal). Esta circunstancia fue especialmente perniciosa, en la medida en que nos obligaba a financiar nuestra creciente deuda pública y déficit comercial con ahorro externo, lo que aumentó nuestra dependencia y vulnerabilidad del exterior en las tormentas financieras de principios de los años noventa. Además, en estos años los Presupuestos Generales del Estado perdieron toda su credibilidad como instrumento vector de la política económica, a la vista de las continuas desviaciones entre los créditos aprobados y los liquidados.

\section{EL CAMBIO DE RUMBO DE LA POLÍTICA ECONÓMICA}

La recesión y su secuela de destrucción de empleo se prolongó hasta mediados de los años noventa, momento en que se produce un giro en la política económica española y se inicia el punto de inflexión en el ciclo económico. La principal base de este nuevo enfoque fue la estabilidad macroeconómica (conseguida a través de la reducción del déficit público y de la inflación), la política de concertación social (que permitirá reducir las principales distorsiones del mercado laboral de forma consensuada con los agentes sociales, o, lo que es lo mismo, sin conflictividad social), la política de liberalización de sectores productivos y privatización de empresas públicas (que, a través de una mayor competencia, incrementó la eficiencia productiva de nuestra economía en sectores clave como el energético, las telecomunicaciones, el suelo, el transporte, los colegios profesionales, etc., al tiempo que se redujeron los precios en la mayor parte de ellos) y, por último, la reducción de la presión fiscal.

El actual ciclo está siendo más estable y prolongado que en épocas anteriores, gracias a la ausencia de desequilibrios que, a la postre, lo acabarían estrangulando. El gran rédito de estos años ha sido nuestra incorporación a la mone- 
da única europea, que, gracias a nuestros esfuerzos de consolidación presupuestaria y de control de precios, nos ha permitido disfrutar de unos niveles de tipos de interés desconocidos en nuestra historia reciente, al tiempo que profundizar en nuestra apertura exterior y en la internacionalización de nuestras empresas. En este sentido, el desarrollo de multinacionales de origen español constituye uno de los principales cambios estructurales de los últimos años, ya que nuestras empresas, por fin, están saliendo del retraso acumulado en las últimas décadas, de forma que hemos pasado de ser receptores a emisores de inversión directa. La economía española ha recogido, en estos años, los frutos de su esfuerzo de convergencia nominal con Europa, sentando así las bases para la creación de empleo y de bienestar. Todo ello demuestra que la estabilidad macroeconómica incentiva la actividad, al promover un patrón de crecimiento saneado que, a su vez, nos está permitiendo avanzar en la convergencia real con nuestros socios europeos.

La recuperación económica que se inició a partir de 1996 se apoyó, en un primer momento, en el dinamismo de nuestro sector exportador, beneficiándose de los efectos positivos de nuestra apertura exterior, así como de la mejora de nuestra competitividad, gracias al cambio de actitud que supuso una mayor atención a factores como la calidad o la evolución de los precios internos, en lugar del tipo de cambio (Requeijo, 2000). A estos factores se une la moderación de los costes financieros y salariales, tras un proceso de fuerte reestructuración empresarial, que permitió mejorar la productividad de nuestras empresas y su posición competitiva frente al exterior. A medida que mejoraban las expectativas de los agentes económicos, la demanda interna fue sustituyendo al sector exterior como motor del crecimiento, en primer lugar la inversión productiva $y$, posteriormente, el consumo privado. De esta forma, la excelente evolución del empleo y su mayor estabilidad han reducido la incertidumbre de las familias, al tiempo que ha incrementado la confianza sobre la sostenibilidad futura del ciclo. Ello, junto con la reducción de los tipos de interés y la mayor renta disponible, gracias a las rebajas de impuestos, han alentado el consumo privado. Por su parte, el tirón de la demanda interna, junto con los menores tipos de interés y la contención salarial, han permitido a las empresas incrementar sus beneficios y aumentar la utilización de su capacidad productiva, lo que explica el notable impulso de la inversión.

La consolidación fiscal ha logrado reducir el déficit público desde el 7,3 por 100 del PIB en 1995 hasta el equilibrio presupuestario previsto para este año, gracias a la implantación de una cultura de rigor presupuestario. Para ello, se introdujeron mecanismos de control del gasto (como fueron la reducción de los créditos ampliables, el control mensual del gasto en la Seguridad Social y los Organismos Autónomos, la reforma de la Ley General Presupuestaria y la creación de la Oficina Presupuestaria y de la Secretaría de Estado de Presupuestos), con el fin de devolver a los Presupuestos la credibilidad perdida en años anteriores, caracterizados por su incumplimiento sistemático. En este sentido, se ha llevado a cabo una política de racionalización del gasto en empleo 
público basada no sólo en la reducción de efectivos, sino también en el estímulo de la productividad de los ya existentes para contener los costes laborales unitarios. De forma pareja a la recuperación económica y al cumplimiento de los criterios de convergencia nominal, asistimos a una reducción considerable de los gastos por prestaciones de desempleo y de los gastos financieros, lo que generó un indudable alivio en los pagos de la carga de la deuda pública, favoreciendo el proceso de consolidación fiscal.

El recorte del gasto se ha producido, principalmente, en los gastos corrientes, salvando aquellas partidas que inciden más directamente en la actividad y en la competitividad (infraestructuras, I+D+i, educación), tras unos años en que se sacrificaron las partidas de inversión. Así, en 1997, por vez primera desde 1992, el déficit público cumplió la regla de oro de las finanzas públicas, al financiar la inversión y la dotación de infraestructuras, y no el gasto corriente (Velarde, 1998). Para promover la innovación e impulsar el gasto en investigación y desarrollo, se mejoró el marco legal que protege los resultados obtenidos de la investigación tecnológica y se establecieron incentivos fiscales para la innovación, incorporados en el Plan Nacional de Investigación Científica, Desarrollo e Innovación Tecnológica, con el objetivo de situar en el 1,3 por 100 del PIB el esfuerzo inversor en $\mathrm{I}+\mathrm{D}+\mathrm{i}$ en el año 2003. Prueba de esta apuesta decidida por la innovación y la mejora de la capacidad tecnológica de nuestras empresas es el creciente peso de estas partidas en los Presupuestos, con lo que se pretende recortar nuestro déficit tecnológico con respecto a nuestros socios europeos. En esta línea de incrementar la competitividad de nuestra economía y avanzar en la convergencia real, se ha puesto en marcha un Plan de Infraestructuras para el período 2000-2007, financiado con fondos comunitarios y aportaciones públicas y privadas, que incluye actuaciones en carreteras (completando la red de alta capacidad), aeropuertos (mediante la creación de nuevas infraestructuras ante el incremento del tráfico aéreo), ferrocarriles (extendiendo la red de alta velocidad), puertos y telecomunicaciones (con el fin de reducir los desequilibrios existentes en las zonas rurales y las CC.AA. menos desarrolladas).

El gasto social y autonómico, que han sido las dos principales partidas de gasto por volumen, también se han moderado. Por una parte, la Seguridad Social se ha sometido a un necesario proceso de saneamiento (que se instrumentó con la Ley sobre Consolidación y Racionalización del Sistema de Seguridad Social de julio de 1997, fundamentada en las recomendaciones del Pacto de Toledo), con el fin de asegurar la viabilidad del sistema de protección social, mediante la separación de las fuentes de financiación y la lucha contra el fraude (especialmente en las prestaciones de invalidez), pero sin dejar al margen las cuestiones relativas a la mejora de la equidad del sistema. En cuanto a la sanidad se refiere, se ha creado la figura de las fundaciones públicas, introduciendo, así, criterios empresariales en la gestión de los servicios sanitarios. El gasto sanitario también ha tratado de racionalizarse en la partida de farmacia, a través de la introducción de fármacos genéricos, sistemas de precios de referencia, 
reducción del número de productos farmacéuticos incluidos en las listas de la Seguridad Social y reducción de los márgenes comerciales de los farmacéuticos y mayoristas. Por su parte, la descentralización del Estado ha sido uno de los principales motores del gasto público en España, por la ausencia de corresponsabilidad fiscal, ya que, a pesar de la descentralización del gasto, no se dotó a las Administraciones territoriales de un sistema potente de ingresos propios sobre el que tuvieran capacidad normativa: en lugar de ello, su financiación descansó en una participación en los ingresos del Estado. Ante estos problemas, el nuevo sistema de financiación autonómica que ha entrado en vigor este año, así como el acuerdo de financiación local que comenzará a aplicarse en el 2003, dota a las Administraciones territoriales de una mayor corresponsabilidad fiscal y estabilidad financiera.

La consolidación presupuestaria ha permitido reducir la presión fiscal estructural, lo que ha incidido positivamente sobre la competitividad y la iniciativa privada. En este sentido, las reformas del IRPF han avanzado en la dirección correcta, ya que se ha incrementado la renta disponible de las familias y de los pequeños empresarios y se ha impulsado el ahorro, el trabajo y la asunción de riesgos. El nuevo impuesto supone un cambio radical respecto a la filosofía imperante en los años anteriores, centrada en objetivos recaudatorios y ajena a los efectos perversos que, sobre la actividad económica y el fraude fiscal, generaban unos tipos impositivos excesivos. De ahí que haya sido necesario reducir tanto los tipos marginales como los tramos del impuesto, reduciendo el exceso de carga del impuesto y mejorando su neutralidad y, con ello, la competitividad de nuestro tejido productivo. Especialmente acertado fue el cambio de referencia imponible desde la renta percibida a la renta disponible, obtenida tras descontar los mínimos personal y familiar, que, por regla general, son más generosos que los anteriores mínimos exentos y deducciones familiares. La reforma impositiva ha permitido dinamizar la actividad económica sin suponer una merma de ingresos para las cuentas públicas, sino todo lo contrario. En este sentido, ha supuesto un claro avance en nuestra adecuación al nuevo entorno caracterizado por la globalización, en el que nuestra mejora de renta y bienestar dependen de la competitividad de nuestro sistema productivo.

En cuanto a la política de rentas, la moderación salarial ha sido fundamental para el control de la inflación, y ha sido posible, en buena medida, gracias al consenso social. Fruto del mismo fue la reforma laboral de 1998, que supuso un avance decidido hacia la flexibilización de nuestro mercado laboral, basándose en el fomento del empleo estable (reduciendo la elevada temporalidad provocada por los altos costes de despido y, con ello, la segmentación del mercado de trabajo) y la mejora del sistema de negociación colectiva, dotándolo de mayor flexibilidad y enriqueciendo su contenido (OCDE, 1998). Por otro lado, las medidas adoptadas en 1998 para incentivar la contratación a tiempo parcial trataron de introducir una mayor flexibilidad en las relaciones laborales, con el fin de adaptarse a las necesidades de determinadas actividades o colectivos. Por su parte, ha sido acertado rechazar la reducción por ley de la 
jornada laboral, puesto que sería perjudicial en términos de inflación y empleo y conduciría a un incremento de los costes empresariales, a una reducción de la producción y del poder adquisitivo, así como a una mayor carga para las finanzas públicas. Queda pendiente, sin embargo, una reducción en mayor profundidad de las cotizaciones sociales (especialmente a cargo de los empresarios). En definitiva, la evolución del empleo en estos años ha sido muy positiva, gracias a la fortaleza de nuestra economía, a la moderación salarial y a los mayores beneficios empresariales, junto con las reformas laborales que no sólo han permitido flexibilizar el mercado de trabajo, sino también aumentar la estabilidad en el empleo.

En otro orden de cosas, las medidas liberalizadoras han apostado por la economía de mercado y por la reforma y modernización del sector público en aras de incrementar su eficiencia, fijándose el contenido ordenado y explícito del programa de privatizaciones. Lo más novedoso de esta nueva etapa es que la política de privatizaciones se configura paralelamente a una política de desregulación, que se concreta en medidas legislativas tendentes a fomentar y liberalizar la actividad económica, y a una política de neorregulación (De la Cruz, 1999), con la que se pretende introducir un nuevo marco de relaciones entre el Estado y las empresas reguladas, con el fin de lograr mayores dosis de competencia en los sectores afectados. Las primeras medidas, introducidas en junio de 1996, orientadas a incrementar la competencia en los mercados, fueron la desregulación o la eliminación de restricciones en algunos mercados. Desde entonces, se han ido materializando los diferentes programas impulsores del proceso de neorregulación en los sectores estratégicos españoles:

- La reforma de la Ley del Suelo de abril de 1998 incluía, como principal novedad, un nuevo concepto de suelo urbanizable, limitando la discrecionalidad de los Ayuntamientos e introduciendo mayor flexibilidad en el desarrollo del suelo urbanizable. Asimismo, se retomaron los valores reales en las expropiaciones, en lugar de los valores confiscatorios, basados en razones de justicia (valor de mercado y no un valor ficticio fijado por la Administración) y equidad (mediante la igualdad de criterio para todos los suelos) (Nasarre, 1997). Finalmente, se devolvió la condición de subsidiariedad a la actuación pública frente a la iniciativa privada y la libertad de empresa.

- Dentro de las telecomunicaciones, se creó la Comisión del Mercado de las Telecomunicaciones, encargada de velar por el buen funcionamiento del mercado; se suprimió el monopolio del servicio telefónico, se flexibilizó la restricción a la entrada de inversión extranjera y se permitió la entrada de competidores de carácter local. La Ley General de Telecomunicaciones de abril de 1998 inició el proceso desregulador en el sector, facilitando la interconexión de las redes en igualdad de condiciones para todos los operadores.

- La Ley del Sector Eléctrico de noviembre de 1997 introdujo la libertad 
de instalación, mediante un mercado mayorista de generación (tipo pool) y uno minorista de provisión (se liberaliza el transporte y la distribución, facilitando el acceso de terceros a las redes), que sustituye, así, al Estado como único agente responsable tanto de la planificación como de la explotación. El objetivo continúa siendo garantizar un suministro de calidad, pero de forma económica, al considerarse el coste de la energía como un factor clave de la competitividad empresarial. El papel del Estado quedó limitado a la planificación de las instalaciones de transporte, así como a la orientación y asesoramiento de las inversiones de los agentes privados.

- En el sector minero, la modernización y reforma de nuestras estructuras productivas hacían necesario poner fin a las ayudas públicas injustificadas que habían mantenido estas actividades ineficientes. En julio de 1997 se aprobó un Plan encaminado a recortar tanto la producción como las plantillas, de acuerdo al calendario fijado por la Comisión Europea para eliminar las ayudas a este sector.

- La Ley de Hidrocarburos de 1998 profundizó en la senda iniciada en septiembre de 1996, cuando se liberalizó el acceso de terceros a la red de transporte para los grandes consumidores industriales. Aunque el almacenamiento estratégico, el transporte y la distribución están sujetos a regulación (no así la comercialización), todas las actividades se desarrollan en régimen de competencia. Los precios se liberalizarán progresivamente hasta el año 2008, mediante el paulatino aumento de los consumidores cualificados (que pueden obtener el gas directamente o de los comercializadores) y el libre acceso de terceros a las instalaciones.

- Otros sectores también han registrado cambios en su regulación: régimen de farmacias (mediante la libertad de horario y la reducción de los márgenes comerciales y de los almacenes), servicios públicos municipales (como el servicio funerario), así como los colegios profesionales (estableciendo la colegiación única en todo el territorio y eliminando los honorarios mínimos), servicio postal, tabaco (a través de la liberalización de la producción, importación y distribución), etc.

Por lo que respecta al programa de privatizaciones, el punto de partida fue el Acuerdo de Modernización del Sector Público Empresarial de 1996, por el cual se encomienda a la Sociedad Estatal de Participaciones Industriales (SEPI) vender las empresas rentables de la antigua Teneo (disuelta en junio de 1996): Repsol, Endesa, Inespal o Iberia, mientras las empresas no rentables (siderurgia, minería o astilleros) formaban la Agencia Industrial del Estado (AIE). Estas últimas fueron transferidas a la SEPI en septiembre de 1997, mientras que las empresas dependientes de la Dirección General del Patrimonio y Ministerio de Economía (como Tabacalera o Argentaria) pasaron a depender de la Sociedad Estatal de Participaciones Patrimoniales (SEPPA). Asimismo, se creó un Consejo Consultivo de Privatizaciones, encargado de garantizar que 
los procesos privatizadores se llevaran a cabo con transparencia y de forma eficiente. Este proceso ha supuesto un traspaso de poder y rentas desde el sector público al privado que, no obstante, no ha provocado una concentración en manos privadas. Todo lo contrario, el proceso de privatizaciones, junto con la liberalización de mercados, ha introducido mayor competencia en nuestra economía, con sus consiguientes efectos positivos sobre la eficiencia empresarial y ganancias de bienestar para los consumidores, en términos de menores precios y mayor calidad y variedad de los bienes ofertados. Al mismo tiempo, los ingresos procedentes de estas ventas han permitido sanear empresas deficitarias, acometer el pago de jubilaciones comprometidas con trabajadores afectados por la reconversión o cancelar deuda histórica contraída por el antiguo INI. Finalmente, las privatizaciones acometidas en los últimos años han favorecido la aparición de un capitalismo popular, con los efectos positivos que ello conlleva de configuración de una cultura financiera y estímulo al ahorro.

En conclusión, la modernización de la economía española nos coloca en una situación inmejorable para afrontar momentos delicados como los actuales, pero ello no habría sido posible sin un importante esfuerzo, puesto que la obtención de un crecimiento elevado, duradero y estable pasa ineludiblemente por lograr la máxima estabilidad y flexibilidad de nuestra estructura productiva. La apertura exterior en un entorno internacional cada vez más globalizado, junto con la pérdida del tipo de cambio como herramienta de política económica tras nuestra incorporación a la moneda única europea, nos han obligado a mejorar nuestra competitividad, mediante reformas estructurales y liberalización de mercados, por un lado, y la corrección de los desequilibrios fundamentales (déficit público e inflación), por otro. En este sentido, la reorientación del papel del Estado en la economía y, en concreto, de la política fiscal como único instrumento efectivo para lograr la estabilidad macroeconómica, a través del equilibrio presupuestario estructural, ha permitido reducir la presión fiscal y, al mismo tiempo, garantizar el Estado de Bienestar.

\section{BIBLIOGRAFÍA}

Aríztegui, J. (1993): «La política monetaria», en España, economía (J. L. García Delgado, dir.), 6. ${ }^{a}$ ed. aumentada y actualizada, Espasa Calpe, Madrid.

BARCIELA, C. (1994): "Fraude fiscal y mercado negro durante el primer franquismo», Hacienda Pública Española, n. ${ }^{\circ} 1$ (monografía), pp. 367-381.

Carreras, A. (1987): «La industria: atraso y modernización», en La economía española en el siglo XX. Una perspectiva histórica, 2. ${ }^{\mathrm{a}}$ ed., Ariel, Barcelona.

- (1997): «La industrialización: una perspectiva a largo plazo», Papeles de Economía Española, n. ${ }^{\circ} 73$, pp. 35-60.

Comín, F. (1993a): «Estado y crecimiento económico en España: lecciones de la historia», Papeles de Economía Española, n. ${ }^{\circ}$ 57, pp. 32-56.

- (1993b): «Las Administraciones Públicas», en España, economía (J. L. García Delgado, dir.), 6. ${ }^{\text {a }}$ ed. aumentada y actualizada, Espasa Calpe, Madrid.

- (1996): Historia de la Hacienda Pública. II. España (1808-1995), Crítica, Barcelona. 
De LA CRUZ, J. (1999): La liberalización de los servicios públicos y el sector eléctrico. Modelos y análisis de la Ley 54/1997, Marcial Pons, Madrid.

Donges, J. B. (1976): La industrialización en España, Oikos-Tau, Barcelona.

FANJUL, E. (1981): «El papel de la ayuda americana en la economía española, 1951-1957», ICE, n. ${ }^{\circ} 577$, pp. $159-165$.

Fuentes, E. (1984): «El Plan de Estabilización económica de 1959, veinticinco años después», ICE, n. ${ }^{\text {os }}$ 612-613, pp. 25-40.

- (1993): "Tres decenios largos de la economía española en perspectiva», en España, economía (J. L. García Delgado, dir.), 6. a ed. aumentada y actualizada, Espasa Calpe, Madrid.

García Delgado, J. L., y Jiménez, J. C. (1999): «El proceso de modernización económica: perspectiva histórica y comparada», en España, economía: ante el siglo XXI (J. L. García Delgado, dir.), Espasa Calpe, Madrid.

GonZÁlez, M. J. (1977): «La historia económica de España en el período 1939-59. Una interpretación", Moneda y Crédito, n. ${ }^{\circ} 143$.

- (1979): La economía politica del franquismo, 1940-1970. Dirigismo, mercado y planificación, Tecnos, Madrid.

Martín Rodríguez, M. (1993): «Evolución de las disparidades económicas regionales: una perspectiva histórica», en España, economía (J. L. García Delgado, dir.), 6. a ed. aumentada y actualizada, Espasa Calpe, Madrid.

Nasarre, F. (1997): «El Proyecto de Ley estatal del Suelo y la STC», Revista del Instituto de

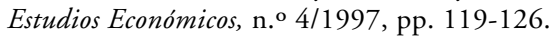

NúNEZ, G. (1998): "La empresa española en el siglo XX», en Historia de la empresa mundial y de España (J. L. García Ruiz, coord.), Editorial Síntesis, Madrid.

OCDE (1998): Estudios económicos de la OCDE: España 1997/1998, Mundi-Prensa, Madrid.

PERPIŃÁ, R. (1993): "Corología agrícola y económica general de España. Ordenación espacio temporal de población y estructura de riqueza», ensayo original de 1958 que aparece en $D e$ economia hispana y otros ensayos, FIES, Madrid, pp. 75-178.

Portillo, M. J. (1998): "La flexibilidad tributaria en España entre dos reformas tributarias: 1940-1957», Hacienda Pública Española, n. ${ }^{\circ}$ 144, pp. 153-173.

Prados de la Escosura, L. (1997): "Política económica liberal y crecimiento en la España contemporánea: un argumento contrafactual», Papeles de Economía Española, n. ${ }^{\circ} 73$, pp. 83-99.

REQUeIJO, J. (1983): «Los circuitos privilegiados de financiación y reforma del crédito oficial», ICE, n. ${ }^{\circ} 596$.

- (1988): Introducción a la Balanza de Pagos de España, Tecnos, Madrid.

- (1990): «Lo que fuimos y lo que somos», ICE, n. ${ }^{\text {os }}$ 676-677, vol. I, pp. 5-18.

- (2000): «El sector exterior español: de los cambios múltiples a la moneda única», en la obra, dirigida y coordinada por J. Velarde, 1900-2000: Historia de un esfuerzo colectivo, Editorial Planeta, Madrid, cap. XI, pp. 583-628.

SEgura, J. (1993): «Intervención pública y política de bienestar: el papel del Estado», en España, economía (J. L. García Delgado, dir.), 6. ${ }^{\text {a }}$ ed. aumentada y actualizada, Espasa Calpe, Madrid.

SERrano, J. M. (1997): «Sector exterior y desarrollo en la economía española contemporánea», Papeles de Economía Española, n. ${ }^{\circ} 73$, pp. 308-335.

Tamames, R. (1982): Estructura Económica de España, 14. ${ }^{a}$ ed., 2 vols., Alianza Universidad, Madrid.

Tortella, G. (1995): El desarrollo de la España contemporánea. Historia económica de los siglos XIX Y XX, Alianza Universidad, Madrid.

Varela, M. (1990): «El Plan de Estabilización como yo lo recuerdo», ICE, n. ${ }^{\text {ss }} 676-677$, vol. I (diciembre 1989-enero 1990), pp. 41-55.

Velarde, J. (1996): Hacia otra economía española, Espasa, Madrid.

- (1998): «El modelo Aznar-Rato», Cuadernos de Formación Veintiuno, Serie Azul, n.o 16.

Velasco, C. (1984): «El ingenierismo como directriz básica de la política económica durante la autarquía (1936-1951)», ICE, n. ${ }^{\circ}$ 606, pp. 97-106. 


\begin{abstract}
Spain has become a modern economy that is fully incorporated into global economic reality and integrated into an ambitious project such as the euro. The huge transformation we have experienced in the last fifty years is, on the one hand, the result of the consolidation of our opening up to the outside world and, in particular, our active participation in the process of the building of Europe and, on the other hand, macroeconomic stability and the protagonism of the market, as a mechanism for allocation of resources, as against traditional public interventionist factors, which dominated a large part of our recent economic history. In short, the road to prosperity, growth and the generation of wealth arises out of integration into world markets and, in particular, into the common European project, with a view to taking advantage of the benefits of globalization over productivity, specialization, optimum growth and the creation of employment.
\end{abstract}

